\title{
Anterior cruciate ligament deficiency: rotational instability in the transverse plane. A preliminary laboratory in vivo study
}

\author{
A. Ferrer ${ }^{1}$, R. Twycross-Lewis ${ }^{1}$, N. Maffulli ${ }^{2,3,4}$
}

1 Queen Mary University of London, Barts and the London School of Medicine and Dentistry, Centre for Sports and Exercise Medicine, Mile End Hospital, London, UK

2 Department of Musculoskeletal Disorders, Faculty of Medicine and Surgery, University of Salerno, Via Allende, Baronissi, Salerno, Italy

${ }_{3}$ Centre for Sports and Exercise Medicine, Barts and The London School of Medicine and Dentistry, Mile End Hospital, 275 Bancroft Road, London E1 4DG, England

4 Institute of Science and Technology in Medicine, Keele University School of Medicine, Stoke on Trent ST4 8FB, England

\section{CORRESPONDING AUTHOR:}

\section{Nicola Maffulli}

Department of Musculoskeletal Disorders

Faculty of Medicine, Surgery and Dentistry

University of Salerno

Via Allende

Baronissi (Salerno)

ITALY

email: n.maffulli@qmul.ac.uk

DOI:

10.32098/mltj.01.2019.17

\begin{abstract}
SUMMARY
Background. Rotational instability is a major feature of anterior cruciate ligament (ACL) deficiency. Few biomechanical studies have focused on the transverse plane when assessing the function of the ACL. Objective. To analyse the biomechanics of ACL deficient knees in the transverse plane under torsional conditions to detect rotational instability. Methods. Seven subjects (eight ACL deficiency) (ACLd group) and nine recreational athletes (controls) were recruited. Each performed two tasks: crossover and pivoting-jump. Biomechanical data were collected in a movement analysis laboratory. Comparisons were made for torque, rotation and torque curves in transverse plane between ACLd, contralateral knees and controls. Results. Torque curves showed and initial avoidance pattern followed by increased values in crossover task, and lesser values for ACLd group compared to controls in pivoting-jump task. Internal rotation in crossover was $19.8^{\circ}, 13.7^{\circ}$ and $19.1^{\circ}$ for ACLd, contralateral and controls respectively $(\mathrm{p}=0.176)$. In pivoting-jump these values were $18.6^{\circ}, 13.8^{\circ}$ and $18.1^{\circ}(\mathrm{p}$ $=0.297$ ). Crossover peak torque were $257,178.4$ and $184.8 \mathrm{Nmm}$ for ACLd, contralateral and controls $(\mathrm{p}=0.5)$, while pivoting-jump values were 238.4, 152.2 and 218 $\mathrm{Nmm}$ for ACLd, contralateral and controls $(\mathrm{p}=0.288)$. Conclusion. Torque curve analysis identified a distinctive pattern differentiating ACLd and control group. However, no statistically significant differences were found in peak transverse plane parameters between groups.
\end{abstract}

\section{KEY WORDS}

anterior cruciate ligament injury; kinematics; kinetics; rotational instability

\section{BACKGROUND}

Anterior cruciate ligament (ACL) tears are common sportsrelated non-contact injuries (1). ACL deficiency can lead to knee instability and secondary joint damage (2). The management of ACL deficiency aims to restore knee function, and subjective and functional outcomes of surgical treatment have been reported as good and excellent in up to $80 \%$ of patients, with sport return rates near to $50 \%$ in amateur patients $(3,4)$. Therefore, a high percentage of patients may experience suboptimal outcomes after treatment. 
Osteoarthritis may develop after ACL injury or reconstruction in up to $13 \%$ of patients with isolated ACL injuries, and in up to $48 \%$ of patients with combined lesions at 10 years of follow up $(5,6)$.

Persistent rotational instability is associated with poor outcomes, and surgical reconstruction techniques have moved from classical transtibial towards more anatomic reconstructions $(7,8)$, with an increasing interest in reconstruction of the anterolateral structures (9). Restoration of the local anatomy is a cornerstone to optimise clinical and biomechanical outcomes (10). Nevertheless, ACL reconstruction does not necessarily restore normal biomechanics $(11,12)$. This probably reflects the fact that the function of the ACL is complex, as it contributes to both anterior translational and anterolateral rotational stability.

Static translational anterior stability can be assessed clinically and instrumentally with the Lachman test and arthrometry, but rotational stability has been difficult to evaluate objectively (13). The pivot-shift test has the highest specificity, but only moderate sensitivity and reliability, and is highly examiner dependent (14). The pivot shift test involves motion in more than one plane, but evaluations of rotational stability should be performed under weight bearing conditions, thus reflecting real life instability (15-17). Previous biomechanical studies focused on the kinematics and kinetics in the sagittal and coronal planes, but less on transverse plane kinetics (12).

Therefore, the present study analysed both kinetic and kinematic parameters on the transverse plane in ACL deficient knees, assuming that rotational instability of the knee can be assessed under torsional weight-bearing conditions. In this way, we wished to ascertain whether a distinctive pattern in the transverse plane torque in ACL deficient knees under dynamic torsional conditions can be identified.

\section{METHODS}

\section{Ethics}

The University Ethics Committee approved all the procedures described in the present study. Each subject received a detailed information sheet and signed a consent form before participating. This study meets the ethical standards of Muscles, Ligaments and Tendons Journal (18).

\section{Subjects}

A total of 16 subjects were recruited. Seven had a total of eight ACL deficient knees, and constituted the experimental group (ACLd group), with a mean age of 33 years (range 20-41). Five subjects had primary ACL injuries, one subject had a chronic re-injury after a full functional recovery, and one subject had a bilateral injury. There were six non-contact injuries related to football practice, and one contact injury in hockey. All subjects met the following five inclusion criteria: a confirmed ACL injury at magnetic resonance, a minimum of 6 months since the injury, an absence of relevant chondral or meniscal lesion, a completed rehabilitation program, and a positive pivot shift. All ACLd subjects were involved in recreational non-pivoting sports at the moment of testing. The mean IKDC score was 69 (range 56-81).

Nine healthy recreational athletes with a mean age of 26 years (range 23-41) and without history of knee pathology were recruited as a control group.

The demographics details of both groups are reported in table I.

\section{Procedures}

All subjects performed two manoeuvres: a crossover task (19) and a pivoting-jump task (15). All tests were performed at the Human Performance Laboratory of our University.

The crossover task consisted of four strides walking fast towards the force plate, and then turning $90^{\circ}$ over the force plate towards the side of the planted foot, crossing over the swing limb. The planted foot had to remain pointing forward avoiding slipping over the force plate (figure 1). The subjects were instructed to reach the centre of the force plate with the tested foot without changes in speed or stride length.

In the pivoting-jump task, the subject started standing over the force plate with the limb to be tested, following by a knee flexion of $90^{\circ}$ or more, while twisting the trunk forward and towards the same leg. In such way, the oppo-

Table I - Demographics and functional features of ACLd and control group.

\begin{tabular}{llll}
\hline & $\begin{array}{l}\text { ACLd group } \\
(\mathbf{n}=7)\end{array}$ & $\begin{array}{l}\text { Control group } \\
(\mathbf{n}=9)\end{array}$ & p value \\
\hline age (years) & $33.2(20-41)$ & $26.7(23-41)$ & 0.098 \\
\hline gender & $\mathrm{m}=5 ; \mathrm{f}=2$ & $\mathrm{~m}=6 ; \mathrm{f}=2$ & 0.554 \\
\hline weight $(\mathbf{k g})$ & $79.7(63-107)$ & $73.1(68-93)$ & 0.331 \\
\hline height $(\mathbf{c m})$ & $175(168-180)$ & $176(159-185)$ & 0.870 \\
\hline dominance & $\mathrm{r}=7 ; 1=1$ & $\mathrm{r}=6 ; 1=2$ & 0.554 \\
\hline $\begin{array}{l}\text { time since } \\
\text { injury (years) }\end{array}$ & $7(0.5-20)$ & $\mathrm{na}$ & \\
\hline IKDC score & $69(56-81)$ & $97(92-100)$ & $<0.001^{1}$ \\
\hline
\end{tabular}

Age, weight, height, time since injury and IKDC score are given in mean (range).

$\mathrm{Na}$, not applicable; $\mathrm{m}$, male; $\mathrm{f}$, female; $\mathrm{r}$, right; 1 , left.

${ }^{1}$ Significant difference. 
site shoulder should go over the plane of the planted lower limb. From this twisted position, the subjects were asked to jump vertically as high and fast as possible (figure 1).

Both tasks force the knee into internal rotation.

Before the definitive data collection, each subject performed at least 10 training attempts to familiarise themselves with the required tasks, which were performed without pain or discomfort. Before the experimental procedures, all subjects were examined by a fellowship trained sports trauma surgeon to verify the diagnosis of ACL deficiency.

\section{Data collection}

Kinematics data were collected using the Codamotion ${ }^{\circledR} 3 \mathrm{D}$ optoelectronic system (Charnwood Dynamics Ltd., Leicestershire, UK) at the Human Performance Laboratory, Centre for Sports and Exercise Medicine, Queen Mary University of London. Four Coda cx $1^{\circledast}$ cameras aligned in a global coordinate system captured infrared signals at a sampling frequency of $200 \mathrm{~Hz}$. Active markers were placed bilaterally on 13 anatomically defined points: the anterior and posterior iliac spines, the lateral and medial femoral epicondyles, the anterior tibial tuberosity, Gerdy tubercle, medial tibial plateau and lateral malleolus, the lateral aspect of the heel, and the head of the fifth metatarsal. Additional clusters of four markers were placed on the lateral aspect of the thigh and leg, and three additional markers were placed onto the patella (one at the inferior pole and two at the superior pole).

Kinetic data were collected using a ground level force plate (Kistler ${ }^{\circledR}$, Winterthur, Switzerland) at a sampling frequency of $500 \mathrm{~Hz}$. A set of three data collection bouts was made for each subject in each task with both legs, and five subjects of each group were tested in two consecutive weeks for reliability purposes.

\section{Data analysis}

Data extraction and analysis were made using inverse dynamics in Matlab $^{\circledR}$ software (MathWorks ${ }^{\circledR}$, Natick, Massachusetts, USA), version r2009a, using custom routines. The kinematic and kinetic data calculated were knee rotation and torque in the transverse plane. For these purposes, the actual knee transverse plane was extrapolated, thus torque was referred to that plane regardless of the degree of motion in the other planes. The average of the three trials for each task was calculated. In the control group, the average of both sides was also calculated, as no significant differences were found between dominant and non-dominant limbs. For torque curve comparisons, an average of curves in the ACLd and control group was obtained, and the whole stance period was divided into 40 equal time intervals.

\section{Statistics}

Descriptive and inferential statistics were performed using the $\mathrm{IBM}^{\circledR}$ SPSS $^{\circledast}$ Statistics software, version 20. Demographic and anthropometric characteristics between groups were compared with the independent samples t-test for numerical variables, and with the Fisher's exact test for categorical data. Dependant variables were tested for distribution in each group with the Kolmogorov-Smirnov and Shapiro-Wilk tests $(\mathrm{p}>0.2$ and $\mathrm{p}>0.5$, respectively). One-way ANOVA test was used to assess differences between groups (ACLd, contralateral and Control groups). Tukey post hoc test was applied for multiple comparisons. Torque curves comparing the ACLd and control groups were analysed normalising stance time in 40 equal time intervals. A t test was applied for each inter$\mathrm{val}$, and the values obtained were checked with $\mathrm{t}$ tables.
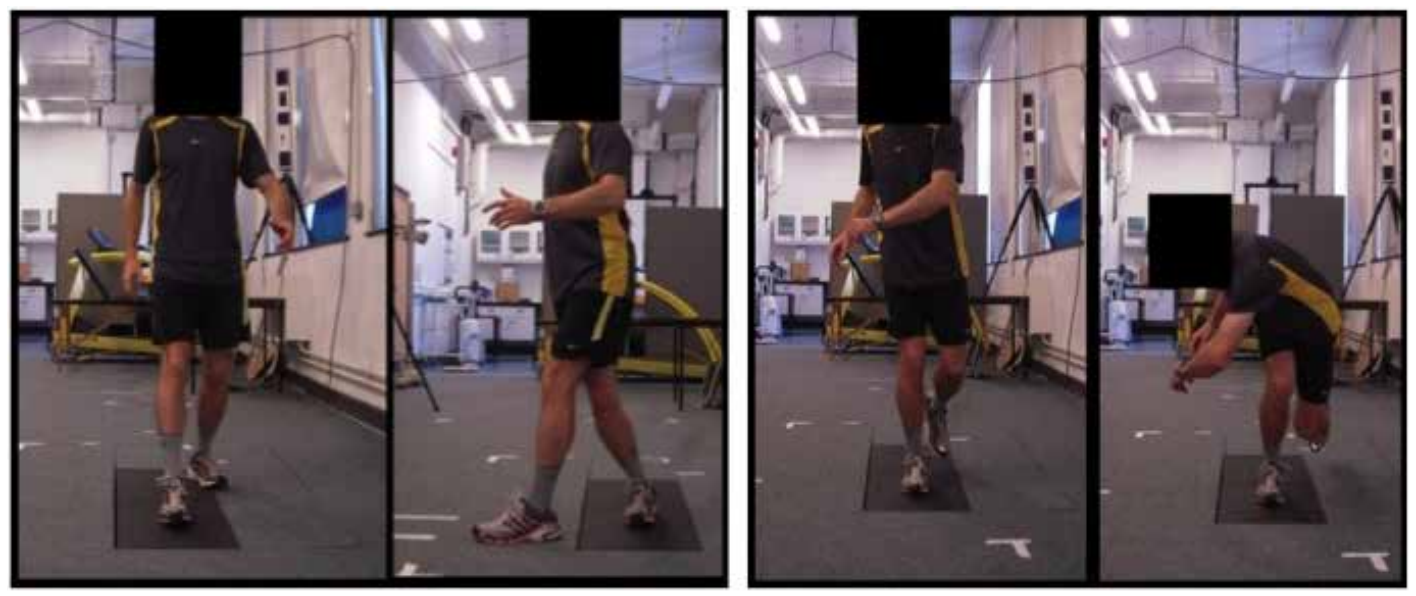

Figure 1 - Left, right-hand side crossover task; right, right-hand side pivoting-jump task. 
Significant difference was set at $\mathrm{p}$ value $<0.05$. Reliability for the dependent variables was tested with the Intraclass Correlation Coefficients (ICC).

\section{RESULTS}

\section{Reliability}

The reliability of tibial internal rotation was good in the pivoting jump task (ICC 0.870), and reasonable in the crossover (ICC 0.703 ). Regarding the internal rotation moment, reliability was good for both the pivoting-jump (ICC 0.757 ) and the crossover task (ICC 0. 808).

\section{Function}

As mentioned above, the mean IKDC subjective score was 69 (range 56-81) in the ACLd group, and 97.2 (range 92-100) in the control group. All ACLd subjects exhibited a positive pivot shift test. Five were classified as $(++)$ and two as $(+)$. No subject in the control group exhibited a positive pivot shift test.

\section{Tibial internal rotation}

\section{Crossover task}

Maximum tibial internal rotation values were not significantly different between ACLd, contralateral and control groups $\left(19.8^{\circ} \pm 7.3^{\circ}, 13.7^{\circ} \pm 5.7^{\circ}\right.$ and $19.1^{\circ} \pm 4.4^{\circ}$, respectively; $\mathrm{p}=0.176$ ).

\section{Pivoting-jump task}

No significant differences were found in tibial internal rotation between ACLd, contralateral and control groups $\left(18.6^{\circ}\right.$ $\pm 9.2^{\circ}, 13.8^{\circ} \pm 6.3^{\circ}$ and $18.1^{\circ} \pm 4.3^{\circ}$, respectively; $\mathrm{p}=0.297$ ).

\section{Torque}

\section{Crossover task}

No significant differences were found in the peak internal rotation torque between ACLd, contralateral and control groups $(257 \pm 152.4,178.4 \pm 129.7$ and $184.8 \pm 83.4 \mathrm{Nmm}$, respectively; $\mathrm{p}=0.5$ ).

\section{Pivoting-jump task}

Peak internal rotation torque values were not significantly different between ACLd, contralateral and control groups $(238.4 \pm 104.4,152.2 \pm 91.8$ and $218 \pm 73.6 \mathrm{Nmm}$, respectively; $\mathrm{p}=0.288$ ).

A summary of biomechanical data is given in table II.

\section{Torque curves}

\section{Crossover}

The knee internal rotation torque during stance was significantly different between ACLd and the control group at three time intervals ( 5 to 8,22 to 29 , and 33 to 39 ), showing the last interval higher values in the ACLd group (figure 2).

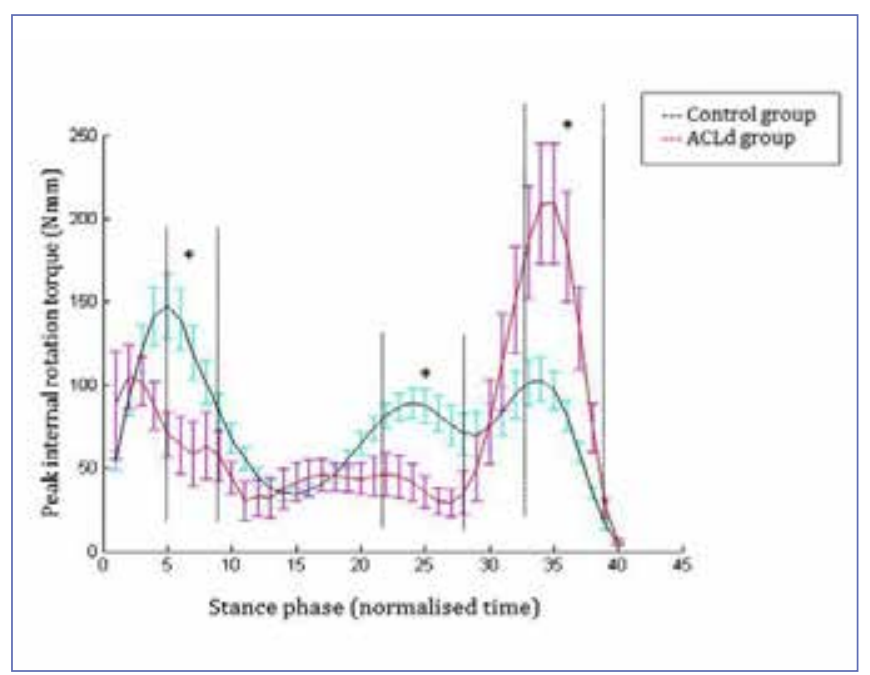

Figure 2 - Internal rotation torque (mean \pm standard deviation) in the ACLd and control group during the stance phase of the crossover task. Black vertical dashed lines with the asterisks show the periods in which the differences were significant.

Table II - Maximum tibial internal rotation and internal rotation torque for each group.

\begin{tabular}{llllll}
\hline & Task & ACLd & Contralateral & Control & p value \\
\hline \multirow{2}{*}{ Internal rotation } & crossover & $19.8 \pm 7.3$ & $13.7 \pm 5.7$ & $19.1 \pm 4.4$ & 0.176 \\
\cline { 2 - 7 } & pivoting & $18.6 \pm 9.2$ & $13.8 \pm 6.3$ & $18.1 \pm 4.3$ & 0.297 \\
\hline \multirow{2}{*}{ Torque } & crossover & $257 \pm 152.4$ & $178.4 \pm 129.7$ & $184.8 \pm 83.4$ & 0.5 \\
\cline { 2 - 7 } & pivoting & $238.4 \pm 104.4$ & $152.2 \pm 91.8$ & $218 \pm 73.6$ & 0.288 \\
\hline
\end{tabular}

Values for internal rotation are in degrees (means \pm SD). Values for torque are in $\mathrm{Nmm}$ (means \pm SD). No significant differences were found. 


\section{Pivoting-jump}

Torque values were lower in the ACLd group across the whole stance period, with significant differences from 13 to 18 time interval (figure $\mathbf{3}$ ).

\section{DISCUSSION}

Torque curves showed significant differences for internal rotation torque between ACLd and the control group at some stance intervals in both tasks, especially in the crossover task.

During the crossover task, the ACLd group showed lower internal rotation torques compared to the control group in the first two thirds of stance, but higher torques toward the end of the manoeuvre. These values can be interpreted as a dual behaviour, in which an initial avoidance pattern (i.e. lower protective values) at heel strike and mid stance is followed by a period of higher load. At this point, maximal torsional stress is transmitted to the knee if the supporting foot stands still until the swing limb completes the $90^{\circ}$ turn. Perhaps it is not possible for ACLd subjects to maintain control or compensate as the crossover approaches to the end.

Remarkably, this increased internal rotation torque was not associated with an increase in internal rotation of the tibia. Therefore, regardless of the increase in the rotating torque acting at the knee, and regardless of the presence of clinical instability, actual dynamic instability was not detected with this manoeuvre.

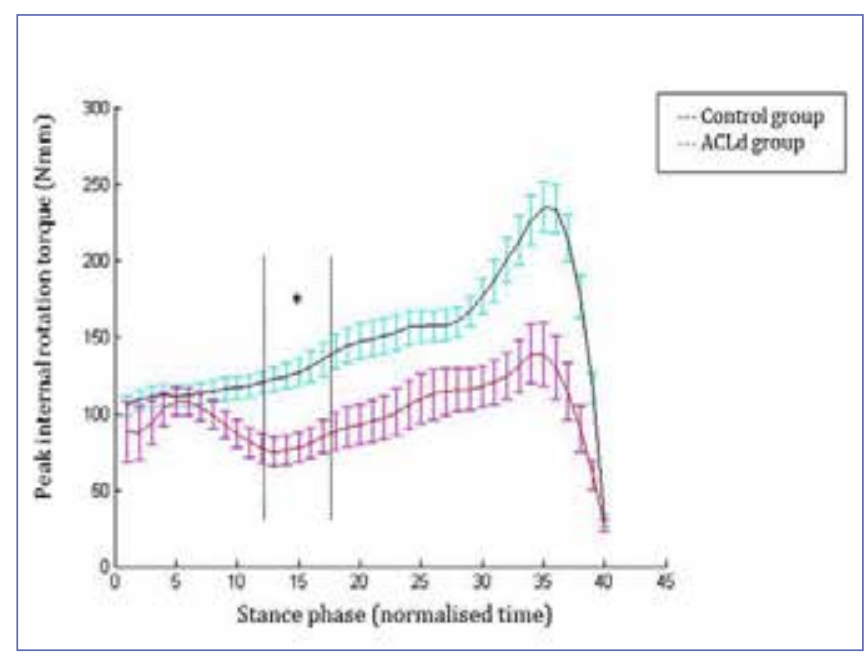

Figure 3 - Internal rotation torque (mean \pm standard deviation) in ACLd and control group during the stance phase of the pivoting task. Black vertical dashed lines with the asterisks show the period in which the differences were significant.
These results are different from other studies which have reported transverse plane parameters in crossover-like tasks. In particular, Houck et al. (16) did not find differences in the transverse plane parameters and curves between ACLd deficient and control groups. However, they showed that compensation occurs at hip level. On the other hand, Tsarouhas et al. (17) reported, using a step-cut manoeuvre task, lower values of internal rotation moment in ACLd knees compared to the contralateral knee and the control group. Our study did evidence higher values of internal rotation moment, thus highlighting a non-avoidance pattern.

The fact that no differences were found in maximum tibial internal rotation could be explained in several ways. First, compensatory mechanisms can be acting to avoid knee rotation, regardless of the amount of rotation torque calculated by inverse dynamics. These compensations may include complex neuromuscular adaptations, which lead to multilevel protective strategies $(20,21)$. Secondly, it is possible that the method used to collect and calculate knee rotation was not sensitive enough. This protocol was used for the first time with marker placement that led to a different segmentation strategy. The use of skin markers can interfere with precise movement measuring. However, the values obtained are quite standard and in line with other studies in this field. Moreover, similar and simpler protocols have shown good sensitivity and reliability to detect tibial rotation (22). Finally, the task itself could be insufficient to produce a detectable increment in internal rotation of the tibia. It is possible that greater internal rotation moments, alone or combined with other force components, are necessary to reproduce rotational instability.

On the other hand, pivoting-jump task, beyond the interval of significant difference, showed a tendency to an avoidance pattern during the whole stance phase. This finding is similar to that reported by Sanchis-Alfonso et al. (15), who found an avoidance pattern in pivoting-jump tasks performed in external and internal rotation. These authors collected only kinetic data, and values of torque were calculated on the force plate surface itself, with the assumption that the knee lies over the point of application of the force, fact that was not proved. Their study concluded that an external rotation-pivoting task was useful to detect such avoidance pattern. This was not the case for the internal rotation task, which was the testing modality used in the present study. However, the authors did not comment on some features of the internal rotation task that were significantly different, such as the impulse, which can be an important variable, as it reflects the time in which the torque is acting. Additionally, as they collected only kinetic data, kinematics (i.e. internal rotation) were not reported. 
The present study could be improved in several aspects. First, the experimental group was not homogenous. Two re-injured subjects were included, but, as they exhibited isolated chronic ACL instability without concomitant injuries, they were considered suitable to assess ACL related rotational instability. Additionally, tasks could be made more standardised to avoid performance bias. The factors that could be controlled are, for instance, speed or actual cutting angle in the crossover task, and knee flexion or trunk torsion in the pivoting jump. Also, the lack of normalization of torque data, more than a weakness itself, is an issue to be considered. In fact, the lesser values of torque were obtained in the heaviest subject, who performed the pivoting-jump task in such way that the ground reaction force passed very close to the centre of the knee. This example reinforces on the one hand the idea of performance standardization, and on the other the fact that the need of normalisation should be evaluated.

Therefore, this study should be considered preliminary, and must inform further investigation involving a greater number of subjects, or perhaps adding different tasks and measurements, so as to identify possible associations between torque applied to the knee and internal rotation of the tibia that could be obtained.

Torque curves can be easily obtained compared with kinematic data collection in a dedicated motion laboratory. Thus, finding a correlation between internal rotation and those curves, we could develop a valid, reliable and time saving method to assess biomechanically in vivo rotational behaviour of ACL deficient or reconstructed knees, as a fine-tuning evaluation of ACL reconstruction.

\section{CONCLUSION}

Given the small size of our cohort, the current study did not identify differences in biomechanical parameters in the transverse plane in the crossover and the pivoting-jump tasks between ACLd, contralateral knee and control groups. Torque curve analysis identified a distinctive pattern in the ACLd group compared to the control group. First, in the crossover task, lower values of internal rotation torque were found at the beginning and mid stance time intervals, followed by higher values toward the end of the stance phase. Secondly, pivoting-jump task showed a tendency towards avoidance pattern. These findings were not associated with increased internal rotation of the tibia. Further work is warranted to improve the quality of the results and stronger conclusions.

\section{Conflict of Interest}

The authors declare that they have no conflict of interest.

\section{Acknowledgements}

We thank Professor Roger Woledge, who recently passed away. This study would not have been possible without his wisdom and generosity. We would like to thank to all the staff of the Centre for Sports and Exercise Medicine, and especially Miss Sue Tracey, for all their support. Dr. Ferrer dedicates this work to his family. Carla and Dante were his permanent inspiration.

\section{REFERENCES}

1. Boden BP, Sheehan FT, Torg JS, Hewett TE. Noncontact anterior cruciate ligament injuries: mechanisms and risk factors. J Am Acad Orthop Surg 2010; 18(9):520-527.

2. Delince P, Ghafil D. Anterior cruciate ligament tears: conservative or surgical treatment? A critical review of the literature. Knee Surg Sports Traumatol Arthrosc 2012; 20(1):48-61.

3. Linko E, Harilainen A, Malmivaara A, Seitsalo S. Surgical versus conservative interventions for anterior cruciate ligament ruptures in adults. Cochrane Database of Systematic Reviews 2005; 18(2).CDO011356.

4. Notarnicola A, Maccagnano G, Barletta F, Ascatigno L, Astuto L, Panella A, et al. Returning to sport after anterior cruciate ligament reconstruction in amateur sports men: a retrospective study. Muscles Ligaments Tendons J 2016; 6(4):486-491.

5. van der Hart CP, van den Bekerom MP, Patt TW. The occurrence of osteoarthritis at a minimum of ten years after reconstruction of the anterior cruciate ligament. J Orthop Surg Res 2008; 24:3612-3618.

6. 6. Oiestad BE, Engebretsen L, Storheim K, Risberg MA. Knee osteoarthritis after anterior cruciate ligament injury: a systematic review. Am J Sports Med 2009; 37(7):1434-43.

7. Franceschi F, Papalia R, Rizzello G, Del Buono A, Maffulli N, Denaro V. Anteromedial portal versus transtibial drilling techniques in anterior cruciate ligament reconstruction: any clinical relevance? A retrospective comparative study. Arthroscopy 2013; 29(8):1330-1337.

8. Ayeni OR, Chahal M, Tran MN, Sprague S. Pivot shift as an outcome measure for ACL reconstruction: a systematic review. Knee Surg Sports Traumatol Arthrosc 2012; 20(4):767-777.

9. Musahl V, Getgood A, Neyret P, Claes S, Burnham J, Batailler C, et al. Contributions of the anterolateral complex and the anterolateral ligament to rotatory knee stability in the setting of ACL Injury: a roundtable discussion. Knee Surg Sports Traumatol Arthrosc 2017; 25(4):997-1008.

10. Bedi A, Musahl V, Steuber V, Kendoff D, Choi D, Allen AA, et al. Transtibial versus anteromedial portal reaming in anterior cruciate ligament reconstruction: an anatomic and biomechanical evaluation of surgical technique. Arthroscopy 2011; 27(3):380-390.

11. Kondo E, Merican AM, Yasuda K, Amis AA. Biomechanical comparison of anatomic double-bundle, anatomic single-bun$\mathrm{dle}$, and nonanatomic single-bundle anterior cruciate ligament reconstructions. Am J Sports Med 2011; 39(2):279-288.

12. Pappas E, Zampeli F, Xergia SA, Georgoulis AD. Lessons learned from the last 20 years of ACL-related in vivo-biome- 
chanics research of the knee joint. Knee Surg Sports Traumatol Arthrosc 2013; 21(4):755-766.

13. Ahlden M, Hoshino Y, Samuelsson K, Araujo P, Musahl V, Karlsson J. Dynamic knee laxity measurement devices. Knee Surg Sports Traumatol Arthrosc 2012; 20(4):621- 632.

14. Benjaminse A, Gokeler A, Van der Schans CP. Clinical Diagnosis of an Anterior Cruciate Ligament Rupture: A Meta-analysis. J Orthop Sports Phys Ther 2006; 36(5):267-288.

15. Sanchis-Alfonso V, Baydal-Bertomeu JM, Castelli A, Montesinos-Berry E, Marin-Roca S, Garrido-Jaen JD. Laboratory evaluation of the pivot-shift phenomenon with use of kinetic analysis: a preliminary study. J Bone Joint Surg Am 2011; 93(13):1256-1267.

16. Houck J, Duncan A, De Haven KE. Knee and Hip Angle and Moment Adaptations During Cutting Tasks in Subjects With Anterior Cruciate Ligament Deficiency Classified as Noncopers. J Orthop Sports Phys Ther 2005; 35(8):531-540.

17. Tsarouhas A, Iosifidis M, Spyropoulos G, Kotzamitelos D, Tsatalas T, Giakas G. Tibial Rotation Under Combined In Vivo Loading After Single- and Double-Bundle Anteri- or Cruciate Ligament Reconstruction. Arthroscopy 2011; 27(12):1654-1662.

18. Padulo J, Oliva F, Frizziero A, Maffulli N. Basic principles and recommendations in clinical and field Science Research: 2016 Update. Muscle Ligament Tendons J 2016; 6(1):1-5.

19. Andrews JR, McLeod WD, Ward T, Howard K. The Cutting Mechanism. Am J Sports Med 1977; 5(3):111-121.

20. Torry MR, Decker MJ, Ellis HB, Shelburne KB, Sterett WI, Steadman JR. Mechanisms of compensating for anterior cruciate ligament deficiency during gait. Med Sci Sports Exerc 2004; 36(8):1403-1142.

21. Markstrom JL, Tengman E, Hager CK. ACL-reconstructed and ACL-deficient individuals show differentiated trunk, hip, and knee kinematics during vertical hops more than 20 years post-injury. Knee Surg Sports Traumatol Arthrosc 2017; 26(2):358-367.

22. Webster KE, McClelland JA, Wittwer JE, Tecklenburg K, Feller JA. Three dimensional motion analysis of within and between day repeatability of tibial rotation during pivoting. The Knee 2010; 17(5):329-333. 